\title{
EVOLUCIÓN CRONOLÓGICA DEL PROCESO DE EXPLOTACIÓN DE ORO EN EL MUNDO \\ Y EN ECUADOR Y SUS EFECTOS SOBRE EL AMBIENTE
}

\section{Chronological Evolution of Gold Exploitation Process In Ecuador And In The World And Its Effects On The Environment}

\author{
Miguel Osorio Rivera*, Teresita Mejía Reinoso, Ángel Flores Orozco, \\ Diana Villa Uvidia, Norma Toledo Castillo, Segundo Vaca Zambrano, Luis Suárez \\ Ávila, Isabel Peñafiel Moncayo, Silvia Salazar Huaraca, Jorge Espinoza Sandoval, \\ Benito Mendoza Trujillo
}

\author{
Escuela Superior Politécnica de Chimborazo (Espoch) \\ extensión Morona Santiago, \\ Don Bosco y José Félix Pintado, Morona Santiago, Ecuador \\ *miguel.osorio@espoch.edu.ec
}

\begin{abstract}
$\mathrm{R}$
El presente trabajo muestra la evolución cronológica de la explotación del oro en el mundo y en Ecuador, partiendo desde la época de la pre-cianuración hasta la denominada fiebre del oro. Se hace énfasis en el aprovechamiento de este recurso que permitió el desarrollo económico de las zonas donde el metal fue explotado y en las consecuencias tanto sociales como ambientales desencadenadas por esta actividad. Además, se describen las técnicas de explotación y refinación, utilizadas para la recuperación de este metal, enfocándose en las técnicas de fito-extracción y fito-remediación como alternativas a los procesos físico-químicos. Finalmente, se resalta cómo Ecuador puede contribuir en el desarrollo de tecnologías alternativas para la extracción de oro, a través del estudio de las características de algunas plantas nativas existentes en la región amazónica.
\end{abstract}

Palabras claves: fito-extracción, fito-depuración, aurífera, plantas nativas.

\section{A bstract}

The following paper shows the chronological evolution of gold exploitation in Ecuador and in the world, starting from the pre-cyanidation period to the so-called gold rush. Emphasis is placed on the use of this resource that allowed the economic development of the areas where the metal was exploited and the social and environmental consequences triggered by this activity. In addition, the exploitation and refining techniques, used for the recovery of this metal, focusing on phytoextraction and phytoremediation techniques as alternatives physical-chemical processes, are described. Finally, it is highlighted how Ecuador can contribute to the development of alternative technologies for gold mining, through the study of the characteristics of some native plants existing in the Amazon region.

Keywords: phyto-extraction, phyto-purification, auriferous, native plants. 


\section{INTRODUCCIÓN}

La explotación del oro ha sido considerada en muchas épocas como uno de los temas predominantes en el mundo, no solo por lo que representa económicamente, sino también por las consecuencias sociales y ambientales que ha generado a través de sus diferentes etapas: producción, refinamiento y comercialización.

Desde sus inicios hasta la actualidad la actividad aurífera se ha constituido en el motor de la economía de muchos países, ya que desde la antigüedad se ha visto como el auge del oro ha formado imperios (11) y así mismo cómo el declive de su producción ha provocado la debacle de las zonas donde se asentaban las minas de gran producción, causando un problema social tanto al inicio como al final de la producción minera.

Además, el daño ambiental que esta actividad ha provocado es sumamente alto, ya que los metales pesados utilizados en el proceso de refinamiento (mercurio y cianuro) cambian drásticamente no solo las condiciones del suelo y de los cauces, si no también afectan directamente a las personas que habitan cerca de zonas mineras, evidenciándose a lo largo del tiempo la presencia de enfermedades relacionadas con la acumulación de dichos metales.

Esta realidad es mundial, pero se acentúa aún más en países de Latinoamérica donde el cumplimiento de la legislación es limitado y sobre todo donde los organismos de control no tienen las capacidades que permitan un adecuado manejo de esta actividad en sus diferentes etapas.

Ecuador, al igual que muchos países de la región, tiene un gran potencial minero, históricamente las primeras provincias dedicadas a esta actividad de forma artesanal fueron Manabí, Esmeraldas, Azuay y Cañar (2). Con la llegada de los españoles en 1492, se comenzó la actividad minería a mayor escala y ya con la fiebre del oro se produjo la industrialización en el país con las consecuencias mencionadas anteriormente.

Los efectos sociales y ambientales generados por esta actividad, han hecho que países como Brasil y Colombia trabajen en nuevas alternativas de refinación del oro, a partir de la riqueza natural de cada país, es decir mediante la utilización de plantas nativas, las mismas que en pruebas preliminares muestran un gran porcentaje de recuperación de oro, respecto a las técnicas tradiciones de mercurio y cianuro (3).

El objetivo de este artículo es brindar una visión amplia de cómo la actividad aurífera ha influido en la historia de los principales países dedicados a esta actividad, tanto a nivel económico, social y ambiental. Además, analiza las técnicas que actualmente se están usando para la recuperación del oro haciendo énfasis en las técnicas alternativas como la fito-recuperación, es decir a través de la utilización de plantas nativas.

\section{RESULTADOS Y DISCUSIÓN}

\section{Evolución histórica de la producción de oro a nivel mundial}

El oro posiblemente fue el primer metal que llamó la atención del ser humano, no solo por su gran belleza en estado nativo sino también porque a diferencia de otros metales, el oro no pierde su brillo en contacto con el aire, es relativamente blando por lo que puede trabajarse fácilmente con martillo, propiedad que les permitió hacer adornos, brazaletes, anillos y collares que se hallaron como restos en las tumbas del hombre neolítico.

Aunque el oro haya sido el primer metal que atrajo la atención del hombre, su utilización aún para ornamentos de carácter simple no fue alcanzada, hasta que el arte de fundirlo fuera inventado en la edad de bronce. De todos modos el oro ha influido favorablemente en el progreso de la civilización pues el afán de poseerlo ha llevado al hombre a explotar y colonizar territorios.

Para comprender el efecto que tiene la producción minera del oro es necesario remontarse al origen de su explotación a nivel mundial, partiendo de la Era de la precianuración hasta la conocida Fiebre del oro.

En Egipto 3050 a.C., el oro era utilizado como moneda de pago en formas de granos y pequeñas barras, se utilizaba también en la decoración de los templos y en la elaboración de joyas. En Mesopotamia cerca de 2700 a.C. el oro era utilizado ornamentalmente, para esto los mineros usaban vellones de oveja en compuertas para 
atrapar oro, se frotaba aceites en el vellón para ayudar a recuperar el oro, lo que aumentaba la eficiencia de esta técnica (1). Después se conoció que una de las aplicaciones más tempranas de la química en el procesamiento de minerales, es el oro humedecido con mercurio (amalgamación) aproximadamente descubierta por el año 1000 a.C., aunque no se usó comúnmente como un proceso comercial de recuperación de oro hasta mucho más tarde. En Turquía alrededor del año 700 a.C. se produjeron las primeras monedas de oro, pero fue solo después de que se desarrolló un proceso de refinación de oro en el año 560 a.C. que se acuñaron monedas de oro puro (5).

A inicios del siglo I d.C. surge el desarrollo de esta actividad en Europa, el Imperio Romano aprovecha la riqueza de este mineral utilizándolo como principal forma de pago para las importaciones, especialmente de China a partir del siglo IV d.C. Los romanos usaban una técnica de descarga por la cual se lavaban las rocas rotas a través de canales que contenían arbustos espinosos, que atrapaban el oro. En España, la minería estaba particularmente bien desarrollada, con nuevas tecnologías como la minería hidráulica, las ruedas de agua y el tornillo de Arquímedes. En este momento también existían prósperas escuelas mineras en toda Europa que proporcionaban la experiencia para la aplicación de nuevas tecnología de extracción (5).

El declive del Imperio Romano dio como resultado una actividad minera disminuida hasta un renacimiento en el siglo XI, con sede en Europa Central. Los desarrollos mineros se centraron en Harz, que ahora es parte de Alemania Oriental y en los Alpes (4). Los procesos de amalgamación y retorta de 1400AD fueron ampliamente utilizados en la extracción de oro.

La prosperidad de las minas de oro y plata centroeuropeas llegó a un abrupto final en la década de 1550, cuando México y las partes de Sudamérica ahora conocidas como Colombia, Perú y Bolivia fueron conquistados por España y las prácticas metalúrgicas europeas se extendieron a Sudamérica. Uno de los primeros hallazgos más significativos fue el de los depósitos aluviales de Chocó en Colombia. Los costos de producción eran más bajos que los de las minas europeas, incluso las distancias de transporte a Europa, en gran parte debido a que las operaciones en América del Sur utilizaban el barrido primitivo, barato y laborioso de los medidores de lecho. En 1693 los depósitos de Minas Gerais en Brasil fueron descubiertos y han estado en producción hasta nuestros días $(5,55,56)$. Una curiosidad de esta época conquistadora es que los metalúrgicos españoles en América del Sur encontraron impuro el oro colombiano, lo mismo que ocurrió con el platino, un elemento desconocido en Europa en ese momento. Se pensó que este material era oro "no maduro" y el tesoro español ordenó que se descartara en el mar como residuo (1).

La competencia sudamericana deprimió la industria minera europea hasta la revolución industrial en Gran Bretaña en el siglo XIX. En Europa durante este período, una técnica común de recuperación de oro fue la amalgamación con placas de cobre. El oro también se produjo a partir de otras fuentes de exploración europea, como África Occidental, que produjo 1 millón de onzas troy para exportar a Europa entre 1400 y 1600 (1). En la primera mitad del siglo XIX, se dio la conocida era de la fiebre del oro, Rusia fue la principal fuente de oro, suministrando el $60 \%$ de la producción mundial. La minería de oro subterránea comenzó en 1744 cerca de Ekaterinburgo, y aumentó después del descubrimiento y el desarrollo de numerosos depósitos aluviales cercanos. Un descubrimiento en Siberia en 1838 en el río Ulderey resultó en una fiebre del oro. Desde 1846, también se produjo una importante actividad minera en la cuenca de Lena. El principal método de recuperación de oro fue la primitiva concentración de gravedad mediante el barrido aunque también se introdujeron trommels y strakes impulsados por vapor y agua.

La producción de oro de Rusia fue eclipsada por una serie de juncos de oro en California (Estados Unidos), Sudamérica, Victoria (Australia) y Nueva Zelanda a mediados del siglo XIX. La fiebre del oro de 1848 en California tras el descubrimiento de oro en el año anterior, fue tal vez la más importante de ellas, abriendo las estadísticas del oeste de los Estados Unidos y contribuyendo significativamente al establecimiento de la nación. Los primeros mineros de California encontraron oro en los lechos de los ríos secos, donde el grado era tan alto que el procesamiento consistía simplemente en arrojar arena y grava en una 
manta: concentración bruta de gravedad seca. Las técnicas de barrido fueron comunes, pero fueron reemplazadas por equipos mejorados de concentración de gravedad húmeda, como cunas y tomas largas, que consistían en pantallas y esclusas. Depósitos aluviales similares fueron explotados en Alaska, Colorado Idaho, Montana, Nevada y Dakota del Sur en los Estados Unidos, y Columbia Británica en Canadá. Sin embargo, las reservas más económicas y fáciles de explotar estaban agotadas, y la importancia de la minería de la veta de cuarzo de roca dura aumentó rápidamente (5).

La fiebre del oro australiana comenzó en 1851 con un hallazgo inicial en Bathurst, New South Wales, y posteriores descubrimientos más grandes cerca de Ballarat y Bendigo en Victoria (1).

Durante la época de la fiebre del oro, el equipo de concentración por gravedad se desarrolló para tratar una gama más amplia de tipos de mineral en escalas progresivamente más grandes. Los circuitos de amalgamación se modificaron para incluir el uso de cianuro de potasio para limpiar el mercurio y la superficie de oro. La concentración de gravedad y la amalgamación se usaron en circuitos de trituración para recuperar oro en la etapa más temprana posible del diagrama - un principio del diseño del diagrama de flujo que aún es válido (1).

\section{Explotación del oro en Ecuador}

El trabajo minero empezó en el país mucho antes de la conquista española. Los aborígenes americanos ya tenían conocimiento del oro, el cobre y el platino. La actividad minera se ha desarrollado en el Ecuador desde tiempos milenarios, pero solamente a nivel artesanal y de pequeña minería, como podría catalogarse en la actualidad (2).

Antes de la Corona Española y la época de la Colonia, los pobladores americanos, en tierra de lo que hoy es Ecuador, explotaban la obsidiana de Mullumica y las arcillas para la cerámica. Trabajaron el oro, la plata, el cobre y el platino para ornamentos, rituales e intercambio comercial, estos minerales se los extraía de ríos y socavones.

Las culturas indígenas, asentadas en lo que hoy es Manabí, Esmeraldas, Azuay y Cañar, fueron las que desarrollaron la minería con mayor fuerza. En Esmeraldas, por ejemplo, los habitantes de la cultura Tolita trabajaron las máscaras de platino, entre los 500 años antes y 500 años después de Cristo. En Azuay y Cañar, la cultura Tacalzhapa se caracterizó por su habilidad para trabajar metales de oro, plata y cobre. En la misma región, los Cañaris han sido considerados históricamente como los elaboradores de la máscara de oro de Chunucari, encontrada en 1940 en el cantón Sigsig, y que ahora es el emblema del Banco Central del Ecuador $(7,58)$.

Pero la llegada de los españoles a América en 1492 y posteriormente a tierras de Quito, marcó un nuevo escenario para la minería del país. La fiebre del oro posibilitó la fundación de Loja (1548, segunda fundación), Zamora (1549), Jaén (1549), Cuenca (1557), Valladolid (1557) y Sevilla de Oro (1575).

Cuando Ecuador se formó y constituyó como nación, la legislación e institucionalidad ecuatoriana fueron débiles y permisivas con las actividades mineras. Luego de la incipiente pero secular explotación de las conocidas minas de Zaruma y Portovelo en el río Amarillo, a principios del siglo XX, el Estado llegó a un acuerdo con South American Development Company (SADCO), que también creó la Cotopaxi Exploration Company para explotar el yacimiento de Macuchi (8,57-59).

Tras la salida de SADCO de Portovelo, se creó la Compañía Industrial Minera Asociada (CIMA), que trabajo hasta los años 70, dejando la explotación en manos de mineros artesanales y pequeños mineros. Ya en los años 80, se redescubrió Nambija y se conocieron los yacimientos de Ponce Enríquez y Cerro Pelado $(8,37)$.

En la actualidad, el Estado ecuatoriano busca una transformación cualitativa para ese intermitente desarrollo minero, mediante la aprobación de la Ley Reformatoria a la Ley de Minería y otras políticas públicas que garanticen el crecimiento de ese sector, con responsabilidad social y ambiental.

\section{Producción mundial de oro}

La producción mundial de oro para el año 2017 alcanzó un máximo de 3269 toneladas, presentado un ligero aumento con respecto a las 3263 toneladas que se extrajeron en el 2016. Este incremento se le atribuye a la aper- 
tura de nuevas minas en países pequeños como Argentina, Bolivia, Brasil, Chile, Colombia, Ghana, Kazajstán, Malí, México, Marruecos, Uzbekistán, Papua, Filipinas, Tanzania, que han servido para compensar la caída de la producción de oro de los grandes productores en el último trimestre del 2017.

Para el año 2017 la producción de oro se distribuye entre los siguientes países:

China es el principal productor mundial de oro con un total de 440 toneladas, evidenciándose una caída en su producción anual del 9\% con relación al 2016. La disminución en la producción se le atribuye a la adopción de normativas más estricta sobre el control de los vertidos contaminantes y el uso de cianuro, que ha provocado el cierre de diversas minas reduciendo la cifra global.

El segundo lugar entre los grandes productores de oro le corresponde a Australia, con 300 toneladas de oro.

Rusia es el tercer productor mundial de oro, con un total de 255 toneladas, registrando un incremento de su producción en el cuarto trimestre de 2017, debido al comienzo de la explotación de la mina de Natalka, en la región de Magadan, de la que se extrajo el primer doré en diciembre de año pasado.

El cuarto lugar le corresponde a Estados Unidos, con un total de 245 toneladas, si bien registró caídas en su producción, estas se debieron a la comparación con los excelentes resultados obtenidos en el mismo periodo de 2016. En Canadá, el crecimiento del último trimestre fue del $5 \%$, gracias a la entrada en funcionamiento de nuevas minas y a la extracción de mineral de más alta concentración en otras, alcanzado las 180 toneladas.

Países latinoamericanos como Perú, México Brasil y Colombia figuran entre los veinte países con mayor producción de oro para el año 2017. Su producción varía entre 155 y 80 toneladas por año.

\section{Métodos de refinación del Oro}

Es necesario conocer los diferentes métodos de refinación del oro para contextualizar la información que se tiene sobre su explotación.

\section{Uso del mercurio en la minería del oro artesanal y en pequeña escala}

El mercurio se usa para separar y extraer el oro de las rocas o piedras en las que se encuentra. El mercurio se adhiere al oro, formando una amalgama que facilita su separación de la roca, arena u otro material. Luego se calienta la amalgama para que se evapore el mercurio y quede el oro. Se usan varias técnicas que liberan distintas cantidades de mercurio.

\section{Amalgamación de todo el mineral}

En este proceso se añade mercurio a todo el mineral durante la trituración, molienda y lavado. Éste es el uso más contaminante del mercurio. En muchos casos sólo el $10 \%$ del mercurio agregado a un barril o a una batea se combina con el oro para producir la amalgama. El $90 \%$ es sobrante y debe retirarse y reciclarse, o se libera en el medio ambiente (5).

Cuando se amalgama todo el mineral, aparecen altos niveles de mercurio que se propagan en el medio ambiente local y crean graves problemas de salud por exposición, tanto para los mineros como para otras personas. Los estudios realizados en lugares donde se practica la amalgamación de todo el mineral muestran los niveles más altos de mercurio en el suelo, los sedimentos y los peces (5).

\section{Concentración gravimétrica o "cribado"}

El cribado (o concentración gravimétrica) de los materiales que contienen oro es un proceso muy común. El oro se concentra con las partículas más pesadas en la batea, y el agua se lleva las partículas más livianas. Luego se agrega mercurio al concentrado para amalgamar o juntar las partículas finas de oro. Esto es mejor que amalgamar todo el mineral. Entre el $10 \%$ y el $15 \%$ del mercurio que se pierde en la minería del oro artesanal y en pequeña escala es consecuencia de este proceso (6).

\section{Quemado de la amalgama}

Los mineros también calientan la amalgama para recuperar el oro. La amalgama se coloca en una pala o cazo de metal y se quema directamente sobre el fuego, a cielo abierto. Cuando esto se hace sin 
usar una retorta, los vapores de mercurio escapan al aire y son inhalados por los mineros, sus familias y demás personas que se encuentren cerca. Esta práctica produce emisiones atmosféricas de mercurio de alrededor de 300 toneladas métricas anuales en todo el mundo (6). Las retortas pueden capturar el vapor de mercurio, evitando que se libere en la atmósfera y disminuyendo los riesgos para la salud de los mineros, sus familias y sus comunidades. Las retortas constituyen una tecnología relativamente sencilla que permite recuperar gran parte del mercurio evaporado de la amalgama (9).

En un proceso que habría sido eficaz aunque probablemente costoso y potencialmente peligroso, Molesworth en 1891 propuso que el oro podría extraerse de la pirita tostando a temperaturas relativamente bajas con la inyección de oxígeno. La calcina podría amalgamarse, aunque se predijo una lixiviación acuífera de 15 a 30 minutos, con oro recuperado de la solución con carbón vegetal (5).

Las propiedades de varios otros productos químicos para disolver oro y plata se conocían a fines del siglo XIX y principios del siglo $\mathrm{XX}$, incluidas las soluciones de bromo - bromuro, cianuro, tiosulfato y tiourea. También se informó que entre 1900 y 1920, algunos minerales que contenían arsenopirita $\mathrm{y} / \mathrm{u}$ oro telururos se trataron con soluciones de bromuro y cianuro (6).

\section{Piro-metalurgia temprana}

Desde el momento del descubrimiento de la amalgamación, el mercurio rico en oro se replicó para eliminar el mercurio y el oro de esponja resultante se fundió con fundentes para producir la venta de oro. Del mismo modo, los concentrados de gravedad, que a menudo contenían magnetita, ilmenita, cromita y otro mineral pesado, se fundieron con potasa, bórax y nitro para eliminar los contaminantes (9).
A lo largo del siglo XIX, varios minerales y concentrados auríferos de plomo, plata y cobre de alta calidad fueron tratados directamente mediante métodos pirometalúrgicos. Estos incluyen lo siguiente: a) Fusión directa en baño de plomo, b) Fundición directa con fundentes ricos en plomo y c) Fundición con fundentes para producir un mate seguido de la fusión con fundentes ricos en plomo. Los procesos generaron aleaciones de plomo-oro-plata, de manera similar, a la bien conocida técnica analítica de ensayo de fuego, en uso a principios del siglo XXI (9). Aunque la investigación, el desarrollo y la aplicación industrial de nuevas tecnologías (especialmente la lixiviación en pilas) se realizaron durante la década de 1970, la década de 1980 produjo más desarrollos técnicos en extracción de oro que cualquier otro período desde el desarrollo de la cianuración. La principal tecnología de procesos químicos aceptada por la industria incluyó el procesamiento CIP (y el carbono en lixiviación [CIL]), la lixiviación en pilas y depósitos de minerales de baja ley, la electro extracción y la reposición, la oxidación a presión de sulfuros, la oxidación biológica de concentrados y la cianuración (6).

Se han demostrado otros procesos como la oxidación de pilas biológicas, los procesos de oxidación biológica de minerales enteros y la resina en pulpa (RIP) a escala de planta piloto o mediante pequeñas operaciones industriales. Un gran número de técnicas se han mostrado prometedoras en el laboratorio, incluida la lixiviación a presión con diversos oxidantes, sistemas alternativos de lixiviación de oro, oxidación de ácido nítrico y aglomeración de carbón y oro (9).

El gas de cloro se descubrió en 1774 y pronto se convirtió en un producto disponible comercialmente. En 1848 Plattner propuso un proceso para el tratamiento de minerales de oro, que consistía en pasar cloro gaseoso sobre mineral triturado para producir un cloruro de oro soluble que podía disolverse en agua. El oro se precipitó a partir de la solución mediante sulfato ferroso, sulfuro de hidrógeno o carbón vegetal. La cloración se usó por primera vez comercialmente para tratar el mineral Deetken (California) en 1858. A mediados de la década de 1860 se usaron diversos procesos de cloración en los Estados Unidos, Sudáfrica y Australia, a menudo para complementar los circuitos de concentración por gravedad existentes o para tratar concentrados ricos en sulfuros. Estas incluyeron rutas totalmente hidrometalúrgicas en las que se agregó cloro en la fase de solución (5).

La cloración rara vez se aplica directamente a los minerales de lana, principalmente debido al alto costo del tratamiento, que dio como resultado altos grados de corte 
de oro (aproximadamente 50 gr). Además, los minerales que contienen arseniuros, antimoniuros y grandes cantidades de sulfuros debieron oxidarse antes de la cloración, como también lo fue para la cianuración en el próximo siglo (6).

\section{Lixiviación de oro con tiosulfato de sodio}

La lixiviación de oro con tiosulfato de sodio, permite manejar minerales refractarios (24). El tiosulfato en el proceso de lixiviación se utiliza acompañado de catalizadores como el amoníaco acuoso e iones cúpricos, debido a que este no se disuelve tan rápido como lo hace el cianuro $(23,26,29)$.

La disolución de oro se realiza a partir de reacciones redox, que transforman el oro metálico en oro-tiosulfato, mientras que el amoníaco acuoso y los iones cúprico forman el complejo cuprotetramina el cual acelera la disolución del oro $(23,25)$.

La lixiviación con tiosulfato necesita establecer un rango de $\mathrm{pH}$ entre 9.6 -11, para lo cual es importante el amoniaco acuoso $(22,26)$. En este rango de $\mathrm{pH}$ es posible que se forme la cuprotetramina (27). Si el rango de $\mathrm{pH}$ es menor aparecen otros complejos aminos y la cuprotetramina es menor. $(23,27)$.

El complejo cuprotetramina se forma a través de los iones cúpricos y del amoniaco, un incremento en la concentración de iones cúpricos ayuda a una rápida disolución de oro $(22,28)$. Pero el incremento excesivo de iones cúprico puede causar la oxidación del tiosulfato en politionatos, estos causan una disminución en la concentración de tiosulfato $(22,28)$.

La excesiva cantidad de la sal de tiosulfato puede ocasionar la reacción entre el ion tiosulfato y el oro metálico (23). La elevada concentración de tiosulfato de sodio, ocasiona que la disolución de oro ocurra de manera lenta, debido a que se minimiza el efecto de la cuprotetramina (28).

Un problema con esta técnica, por lo cual no ha sido considerada como una técnica industrial, se encuentra en la recuperación del oro contenido (23). Últimamente se ha utilizado la adsorción con resinas ya que el proceso convencional de adsorción con carbón activado es inservible para recuperar oro de los lixiviados (22). Pero el costo de instalación y la necesidad de regenerar las resinas no han permitido que esta técnica sea rentable para la recuperación de oro (23).

\section{Fitorremediación de los desechos de la minería de oro}

La fitorremediación ha recibido una mayor atención en las últimas décadas, como un enfoque emergente y eco- lógico, que utiliza la capacidad acumuladora e hiperacumuladora que poseen algunas plantas para remediar el agua, los suelos o los sedimentos contamina$\operatorname{dos}(3,14)$.

La eficiencia de remoción de contaminantes durante el proceso de fitorremediación dependerá principalmente de la especie de planta utilizada, el estado de crecimiento de las plantas, su estacionalidad y el tipo de metal a remover (18). Siendo la lenteja de agua y el Jacinto de agua las especies más utilizadas.

La lenteja de agua (Lemna minor) comprende un grupo de pequeñas plantas acuáticas de rápido crecimiento, que se caracterizan por formar extensos mantos sobre cuerpos de agua con movimiento léntico; su reproducción generalmente es vegetativa. El contenido de proteína que se reporta oscila entre $13-41 \%$, y depende del contenido de nitrógeno en el medio en el cual se desarrolla (16). Tiene preferencia por el consumo de amonio sobre el nitrato, con una capacidad de absorción de metales pesados entre ellos el mercurio, razón por la cual se ha utilizado para el tratamiento de aguas residuales con excelentes resultados (17). El jacinto de agua (Eichornia crassipes), una planta acuática, de uso ornamental y conocida por su capacidad de producción y la eliminación de contaminantes del agua, ya que absorbe directamente los nutrientes del agua, convirtiendo esta especie en un excelente purificador de aguas residuales. Adaptable a una amplia gama de condiciones ambientales y climáticas. Esta planta es de rápido crecimiento, crece en una amplia variedad de tipos de humedales y prefiere aguas con nutrientes enriquecidos. Sin embargo, se puede tolerar una variación considerable en los niveles de nutrientes, temperatura y $\mathrm{pH}$ (18).

El crecimiento del jacinto de agua se ve significativamente influido por los niveles de nutrientes presentes en el agua, especialmente nitrógeno, fósforo y potasio, además de los principales nutrientes 
también absorben algunos metales pesados. Puede soportar fluctuaciones del nivel de agua, acidez y niveles bajos de nutrientes. No tolera agua salobre y la salinidad puede modificar su distribución (22).

La lenteja de agua (Lemna minor) y jacinto de agua (Eichornia crassipes) han sido usadas en la fitorremediación de aguas residuales contaminadas con mercurio por la actividad minera. Al inicio del experimento se tenía una concentración de 1,2 ppm mercurio $(\mathrm{Hg})$ y al final del experimento se obtuvo una concentración de 0,366 ppm de mercurio $(\mathrm{Hg})$ (15).

\section{Experiencias de uso de guarumbo y malva en la refinación de oro}

Desde el año 2015, la Facultad de Minas de la Universidad Nacional sede Medellín, a través del instituto CIMEX, viene desarrollando investigaciones para proponer una técnica de extracción de oro sin usar mercurio o cianuro.

Como alternativa a dichos metales pesados, se propone el uso de una emulsión natural, obtenida de la trituración de ciertas platas nativas como el yarumo (Cecropia peltata) y el babasaino (Sterculia aerisperma).

El modelo de extracción que se utilizó es similar al método de flotación espumante aplicado principalmente en los yacimientos de cobre y plomo, el mismo que consiste en colocar en una batea la arena que contiene el oro y la emulsión natural y después de una ligera agitación se observa como el oro comienza a separarse de la tierra por flotación. Esta metodología mostró que dicha emulsión tiene la capacidad para sacar a flote entre un 90 y 100 por ciento del oro fino, mientras que el mercurio no lo hace. El oro fino en los yacimientos mineros de Colombia podría estar cercano a un 30 por ciento.

En la actualidad se realizan pruebas químicas de laboratorio para determinar qué cantidad de oro se puede extraer variando las concentraciones de la emulsión natural. Si bien esta investigación ha arrojado valiosos resultados aún no ha sido aplicada en la industria colombiana. A través de esta técnica se utilizan reactivos químicos de bajas concentraciones que no solo mejoran la recuperación, sino también contribuyen con el ambiente al no contaminar los suelos y las aguas superficiales con reactivos fuertes como el mercurio o cianuro.

\section{Problemas socio-ambientales de la actividad aurífera}

El PNUMA (2013) identificó la minería a pequeña escala como la principal fuente antropogénica de mercurio, responsable del 37 por ciento del total de las emisiones antropogénicas en la atmósfera global.

El empleo del mercurio supone un alto riesgo para la salud humana y ambiental, dado que algunas de sus formas y especies químicas tienen una alta toxicidad $(20,43)$. Los impactos de la minería inciden tanto en los recursos hídricos, geológicos, biológicos, atmosféricos como en el ámbito socio-económico y sanitario $(42,44)$.

Las tres vías principales de envenenamiento por $\mathrm{Hg}$ son la inhalatoria, oral y dérmica/ mucosa. La Organización Mundial de la Salud considera aceptable una concentración en el agua de $0,001 \mathrm{mg} / 1 \mathrm{y}$ una ingesta semanal tolerable de $5 \mu \mathrm{g} / \mathrm{kg}$ de $\mathrm{Hg}$ total y 3,3 $\mu \mathrm{g} / \mathrm{kg}$ de $\mathrm{Hg}(21)$.

El ciclo de contaminación por mercurio comienza en los mineros, debido al inevitable contacto con el metal, posteriormente el mercurio es eliminado al ambiente a través de los efluentes líquidos generados en la actividad minera, para finalmente ser transmitido al resto de la población a través de la cadena alimenticia $(45,46)$.

La absorción directa del mercurio gaseoso emanado por incineradores en la extracción del oro induce severas intoxicaciones si se inhala durante periodos prolongados de tiempo $(47,48)$. Los síntomas que generalmente presentan las personas en contacto con este metal van desde la salivación excesiva, dificultad para respirar y fatiga, bronquitis, temblores e irritabilidad, cambios en la personalidad por daños en el cerebro, sensación de dientes que flotan y dolor en los mismos, hasta producir daño renal y respiratorio que puede conducir a la muerte $(20,30,31,40)$.

La falta de tratamiento de los efluentes contaminados por mercurio hace que estos desechos lleguen hasta los ríos y suelos aledaños al área de explotación, provocando lentamente la degradación de los recursos naturales $(54,60)$. Posteriormente la flora y fauna de las fuentes de agua ingieren los residuos de este metal, los peces no mue- 
ren al ingerir residuos de mercurio (39), sin embargo al recibirlo lo transforman en la forma más tóxica de este elemento llamada metilmercurio, que luego afecta a las personas que los consumen, por su propiedad bioacumuladora (20).

El metilmercurio pasa con suma facilidad a través del epitelio digestivo y de allí las membranas biológicas de los demás tejidos del organismo para afectar importantes vías enzimáticas (41). Los riñones y cerebros en el adulto son los principales puntos de concentración; el feto y los recién nacidos son sumamente sensible al metilmercurio $(32,33)$. En neonatos la tasa de mortalidad es elevada y se relaciona de manera directa con la tasa de madres contaminadas $(34,38,53)$.

En los seres humanos, el metilmercurio tiene en promedio una vida biológica media de 70 días en todo el cuerpo. Hay que destacar, que dentro de las cadenas tróficas, el mercurio sufre procesos de bioconcentración, principalmente en los animales marinos y en ciertos productos vegetales, lo que hay que tener muy en cuenta como fuente de contaminación y envenenamiento humano por biomagnificación $(35,36)$. La afectación humana indirectamente se da por la ingestión de peces y agua contaminada con la que se abastece a las poblaciones cercanas $(32,51)$.

En general, la minería provoca una pérdida de tierra para uso agrícola y vivienda, perdida de la biodiversidad, acumulación de metales pesados dentro de la cadena alimenticia, pérdida de agua para uso pecuario, de irrigación o para consumo. En extensos tramos de ríos a nivel mundial, la calidad del agua y de los sedimentos es tal que se ha erradicado toda forma de vida superior, y es imposible el uso benéfico y racional del agua para consumo humano o para irrigación $(49,50,52)$.

Finalmente el trabajo dentro de una mina es riesgoso, pero, aunque son esporádicos los hallazgos representativos del metal, generan ingresos considerables tanto para propietarios como para obreros, la paga supera a las expectativas salariales provinciales e incluso nacionales, por lo que los mineros exponen su salud (11).

\section{CONCLUSIONES Y FUTURAS PERSPECTIVAS}

La mayor parte de la historia mundial es influenciada por la presencia del metal precioso (oro), ya sea para su uso ornamental, joyería o como moneda de intercambio en el pago por servicios. En este sentido, siempre se ha buscado refinar el metal, para recuperar la mayor cantidad posible de oro; es así, que de acuerdo al tipo de refinamiento que se le ha dado al metal también los espacios temporales tenían su nombre propio como: pre-cianuración, amalgamación, cianuración.

De acuerdo a la información revisada, se evidencia que donde existía la explotación de este metal predominaba el poder económico desarrollándose grandes imperios; pero aun cuando existía bonanza, también se evidencia una marcada desigualdad social, puesto que los mineros no obtenían ganancias tan sustanciales como los dueños de las minas o los comercializadores del metal. Una vez que esta bonanza aurífera caía en el volumen de producción estos imperios también caían, generando aún más problemas sociales.

Entonces, este auge del oro a nivel mundial llevó a industrializar el refinamiento del metal, permitiendo recuperar mayor cantidad de oro mediante procesos químicos como la amalgama de mercurio o el humedecimiento con cianuro, los mismos que hasta la fecha son utilizados, en la actualidad el más usado es el segundo método. Los procesos mencionados han provocado deterioro ambiental en las zonas mineras y en sus áreas aledañas, cambiando completamente los ecosistemas. Además, otro problema que se nota es la acumulación de la concentración de estos metales en órganos internos de los mineros causando problemas de cáncer, en cambio en las poblaciones aledañas a estos campos mineros se evidencia mortalidad, ya que utiliza como abastecimiento de agua potable y cultivos, las aguas de ríos que contienen residuos de la minería.

Ecuador no es indiferente a esta realidad, ya que tiene una gran capacidad minera, la misma que desde la llegada de los españoles, su método tradicional de extracción cambio a la forma industrial, ocasionando daños grandes a la naturaleza y sus habitantes. En Ecuador la minería del oro artesanal es una fuente importante de ingresos para los mineros, 
especialmente en comunidades y regiones rurales donde las alternativas económicas son sumamente limitadas. Esta actividad somete a los trabajadores a una grave exposición y crea riesgos para los habitantes de las comunidades cercanas, además se tiene como resultado una extensa degradación del medio ambiente y la contaminación del ecosistema, efectos que pueden perdurar durante varias décadas después del cese de las actividades mineras.

Con el fin de frenar de alguna manera esta contaminación se han buscado varias soluciones, partiendo desde las políticas públicas respecto a la explotación y refinamiento del oro, las mismas que alguna manera regulan no solo la explotación sino el refinamiento y el tratamiento de los efluentes contaminados, pero aún queda un gran trabajo por hacer en la parte artesanal.

Grandes pasos se han dado en el tratamiento químico de los residuos provenientes de esta actividad, pero también se ha utilizado métodos biológicos mediante bacterias o plantas que logran de alguna manera absorber o transformar estos compuestos. Pero además se busca no solo remediar los efluentes que se producen, sino se busca minimizar la contaminación desde el mismo refinamiento, mediante procesos similares a la extracción de plomo y cobre, como es el lavado mediante tensoactivos, o a su vez utilizando plantas nativas que por su composición logran recuperar el metal, realizando el mismo trabajo que realizan los compuestos de cianuro o mercurio. Este tipo de actividades ya se las estudia y practica en Brasil y Colombia, pero en Ecuador aún no se la aplica. Esto se debe a que no existen estudios de este tipo en las zonas mineras, por lo que con el recurso natural que posee el país en flora, sobre todo en plantas similares a las que se usan en Brasil y Colombia, se puede determinar si las plantas nativas de Ecuador también poseen estas cualidades tanto para la refinación como para el tratamiento de efluentes. La Amazonía ecuatoriana cuenta con una gran biodiversidad de flora y fauna, por lo que ofrece un gran campo a explorar, el mismo que aún incierto, pero que presenta un gran potencial de investigación debido a la variedad de plantas que existen en el país y su familiaridad con la de países vecinos donde ya se han realizado estudios. Los esfuerzos deben concentrarse en utilizar especies similares a las adoptadas en otros países para lograr separar el oro y que dicho resultado muestre una diferencia significativa con respecto a los materiales contaminantes utilizados comúnmente para que de esta manera el método pueda ser presentado como una alternativa viable tanto para la extracción, la preservación del medio ambiente y la salvaguardia de la seguridad y salud del minero artesanal. Además debe representar una ventaja económica para que pueda ser rentable tanto a la industria como al artesano.

1. Marsden J, House CI. The Chemistry of Gold Extraction. Segunda ed. Colorado, USA: Society for Mining, Metallurgy, and Exploration, Inc.; 2006. 682 p.

2. Lane K. Unlucky strike: gold and labor in Zaruma, Ecuador, 1699-1820. Colonial Latin American Review. 2004; 13(1): 65-84.

3. Castrillón V, Navarro L. Evaluación de la Fitorremediación como alternativa para el tratamiento de aguas residuales contaminadas con mercurio producto de la minería aurífera (artesanal y pequeña escala) 2017.

4. Collender F. The historical importance of gold in the world's monetary systems. Proceedings Randol Perth International Gold Conference. 1998:383-6.

5. McNulty T. A metallurgical History of gold. American Mining Congress; San Francisco CA1989.

6. Espinoza C, Maldonado A, Veliz M. Análisis comparativo del método ácido con los métodos tradicionales usados en la recuperación de oro en el distrito minero Zaruma-Portovelo. Tesis de grado. Guayaquil: Escuela Superior Politécnica del Litoral, 2014.

7. Sandoval F. La pequeña minería en el Ecuador. IIED and WBCSD (Ed.), Mining, Minerals and Sustainable Development (MMSD). 2001; 75:30. 
8. Paredes D. ¿Después de la minería que?: análisis del impacto socioeconómico y ambiental de la minería: caso South American Development Company (SADCO-CIMA), Portovelo y Zaruma-El Oro-Ecuador. MS thesis. Quito: FLACSO Sede Ecuador, 2013.

9. Ponce M. Investigación Comparativa de los Métodos Clásicos de Refinación de Oro en Eficiencia y Costos, Proceso Agua Regia, Proceso Ácido Nítrico, Proceso Agua Regia sin encuarte, Proceso Outokumpu Modificado, para pequeñas Refinerías de Oro. Tesis de grado. Lima: Universidad Nacional de Ingeniería, 2005.

10. Jiménes R. Introducción a la contaminación de suelos. Madrid, España: Ediciones Paraninfo S.A.; 2017.

11. Spiegel S, Veiga M. Global impacts of mercury supply and demand in small-scale gold mining. Report to the UNEP Governing Council Meeting. 2006.

12. Llugany $\mathrm{M}$, Tolrà $\mathrm{R}$, Poschnrieder $\mathrm{C}$, Barceló J. Hiperacumulación de metales: ¿una ventaja para la planta y para el hombre? Ecosistemas. 2007; 16(2):4-9.

13. Brooks R, Lee J, Reeves R, Jaffré T. Detection of nickeliferous rocks by analysis of herbarium specimens of indicator plants. Journal of Geochemical Exploration. 1977; 7:47-57.

14. Navarro J, Aguilar I, López J. Aspectos bioquímicos y genéticos de la tolerancia y acumulación de metales pesados en plantas. Ecosistemas. 2007; 16(2): 10-25.

15. Jaramillo M, Flores E. Fitorremediación mediante el uso de dos especies Lemma minor (Lenteja de agua), y Eichornia crassipes (Jacinto de agua) en aguas residuales producto de la actividad minera. Cuenca: Universidad Politécnica Saleciana 2012.

16. Zetina P, Reta J, Ortega M, Sánchez M, Herrera, J. Utilización de la lenteja de agua (Lemnaceae) en la producción de tilapia (Oreochromis spp.). Archivos de Zootecnia. 2010; 59:133-155.

17. Arenas A, Marcó LM, Torres G. Evaluación de la planta Lemna minor como biorremediadora de aguas contaminadas con mercurio. Avances en Ciencias e Ingeniería. 2011; 2(3):1-11.

18. Llantoy V, Valderrama A. Estudio de los parámetros fisicoquímicos para la fitorremediación de Cadmio (II) y Mercurio (II) con la especie Eichhornia crassipes (Jacinto de agua). Revista de la Sociedad Química del Perú. 2014; 80(3):164-173.

19. Seccatore J, Marin T, De Tomi G, Veiga M. A practical approach for the management of resources and reserves in Small-Scale Mining. Journal of cleaner production. 2014; 84: 803-808.

20. Vallejo L, García L, Rodríguez M. Percepción sobre daños a la salud y utilidad de medidas de protección de personas expuestas ocupacionalmente al mercurio en la minería del oro. Revista Lasallista de investigación. 2013; 9(1).

21. Cano S. Contaminación con mercurio por la actividad minera. Biomédica. 2012; 32(3): 309-311.

22. Aylmore M, Muir D. Thiosulfate leaching of gold-a review. Minerals Engineering. 2000; 14(2): 135-174.

23. Haddad P, Grosse A, Dicinoski G, Shaw M. Leaching and recovery of gold using ammoniacal thiosulfate leach liquors. Hydrometallurgy. 2003; 69:1-18.

24. Michel D, Delgado E. Lixiviación de minerales de oro con el uso de tiosulfato: tecnología alterna a la cianuración de minerales de oro. RIIGEO. 2011; 13(26):67-72.

25. Navarro P, Villarroel A, Alguacil F. Lixiviación de oro con tiosulfato de amonio catalizado con ion cúprico desde un concentrado polimetálico. Jornadas SAM-CONAMET. 2001; 93-97.

26. Senanayake G. Gold leaching by copper (II) in amoniacal thiosulphate solutions in the presence of additives. Part I: A review of the effect of hard-soft and Lewis acid-base properties and interactions of ions. Hydrometallurgy. 2012; 115-116: 1-20.

27. Senanayake G, Zhang X. Gold leaching by copper (II) in amoniacal thiosulphate solutions in the presence of additives. Part II: Effect of residual $\mathrm{Cu}$ (II), $\mathrm{pH}$ and redox potentials on reactivity of colloidal gold. Hydrometallurgy 2012; 115-116: 21-29.

28. Tsunekawa M, Rath R, Hiroyoshi N, Hirajima T. Ammoniacal thiosulfate leaching of gold ore. Ejmp\&ep. 2003; 3(3): 344-352.

29. Zelinsky A, Novgorodtseva O. EQCM study of dissolution of gold in thiosulphate solutions. Hydrometallurgy. 2013; 138: 79-83. 
30. The International Programme on Chemical Safety. Environmental Health Criteria 118, Inorganic Mercury. Geneva, Switzerland: World Health Organization; 1991.

31. National Research Council. Toxicological Effects of Methylmercury. Washington, DC, USA: National Academy Press 2000.

32. WHO. Mercury. Environmental Health Criteria 1. Geneva: WHO, 1976.

33. Ninomiya T, Imamura K, Kuwahata M, Kindaichi M, Susa M, Ekino S. Reappraisal of somatosensory disorders in methylmercury poisoning. Neurotoxicol Teratol. 2005; 27:643-653.

34. Osores F, Grández A, Fernandez J. Mercurio y salud en Madre de Dios, Perú. Acta Med Per 2010; 27(4).

35. Osores F, Humán M, GrándezA. Intoxicación por mercurio en la región de Madre de Dios: un problema de salud pública. Rev Fac Med Hum. 2009; 9(2): 45-52.

36. Goyzuela G, Trigos C. Riesgos de salud pública en el centro poblado minero artesanal La Rinconada (5200 msnm) en Puno, Perú. Rev Perú Med Exp Salud Pública. 2009; 26(1):41-44.

37. Cosio G. Mining work in high altitude. Arch Environ Health. 1969; 19(4): 540-47.

38. Hurtado J, Gonzales G, Steenland K. Mercury exposures in informal gold miners and relatives in southern Peru. Int J Occup Environ Health. 2006; 12(4): 340-45.

39. Gammons C, Slotton D, Gerbrant B, Weight W, Toung C, McNearny R, et al. Mercury concentrations of fish, river water, and sediment in the Río Ramis-Lake Titicaca watershed, Peru. Sci Total Environ. 2006; 368(23): $637-48$.

40. Adams D, Sonne C, Basu N, Dietz R, Nam D, Leifsson P, Jensen A. Mercury contamination in spotted seatrout, Cynoscion nebulosus: an assessment of liver, kidney, blood, and nervous system health. Science of the total environment. 2010; 408(23), 5808-5816

41. Cogua P, Campos N, Duque G. Total mercury and methylmercury concentration in sediment and seston of Cartagena bay, colombian caribbean. Boletín de Investigaciones Marinas y Costeras-INVEMAR. 2012; 41(2), 267-285.

42. Gallarday T. Estudio del impacto ambiental de la extracción aurífera artesanal dentro de la jurisdicción política del distrito de Santa Rosa de Quives, año 2006. Rev. Inst. investig. Fac. minas metal cienc. Geogr. 2006; 9(18), 98-108.

43. Harris H, Pickering I, George G. The chemical form of mercury in fish. Science. 2003; 301(5637): 12031203.

44. Lozada J. Arends E. Aspectos ambientales de los diferentes tipos de minería de oro, desarrollados en la Reserva Forestal Imataca. Revista Forestal Latinoamericana. 2000; 15(27): 81-99.

45. Marrugo J, Ruiz J, Díez S. Relationship between mercury levels in hair and fish consumption in a population living near a hydroelectric tropical dam. Biological trace element research. 2013; 151(2): 187-194.

46. Ogola J, Mitullah W, Omulo M. Impact of gold mining on the environment and human health: a case study in the Migori gold belt, Kenya. Environmental Geochemistry and Health. 2002; 24(2): 141-157.

47. Olivero J, Johnson B, Arguello E. Human exposure to mercury in San Jorge river basin, Colombia (South America). Science of the total environment. 2002; 289(1): 41-47.

48. Malm O. Gold mining as a source of mercury exposure in the Brazilian Amazon. Environmental Research. 1998; 77(2):73-78.

49. Fadini P, Jardim W. Is the Negro River Basin (Amazon) impacted by naturally occurring mercury? Science of the Total Environment. 2001; 275(1-3):71-82.

50. Nevado J, Martín R, Bernardo F, Moreno M, Herculano A, et al. Mercury in the Tapajós River basin, Brazilian Amazon: a review. Environment International. 2010; 36(6):593-608.

51. Roulet M, Lucotte M, Farella N, Serique G, Coelho H, Passos CS, et al. Effects of recent human colonization on the presence of mercury in Amazonian ecosystems. Water, air, and soil pollution.1999; 112(3-4):297-313.

52. Díaz F. Mercurio en la minería del oro: impacto en las fuentes hídricas destinadas para consumo humano. Rev. Salud pública. 2014; 16(6): 947-957. 
53. Solíz M, Maldonado A, Valladares C. Estado nutricional, desarrollo madurativo y sufrimiento mental en niños, niñas y adolescentes de comunidades cercanas al Proyecto megaminero "Fruta del Norte" en comparación con comunidades de minería artesanal. Revista de la Facultad de Ciencias Médicas de la Universidad de Cuenca. 2013; 3(31): 26-37.

54. Betancourt O, Chalen C, Narváez A, Maldonado M. La Pequeña Minería del Oro: impactos en el ambiente y en la salud humana en la cuenca del río Puyango. Quito: FUNSAD; 2002.

55. Sacher W. Megaminería y desposesión en el Sur: un análisis comparativo. Iconos revista de ciencias sociales. 2015; 19(51): 99-116.

56. Sacher W, Acosta A. La Minería a Gran Escala en el Ecuador. 1era ed. Quito: Abya-Yala; 2012.

57. Sanchez L, Leifsen E. Minería a gran escala en Ecuador: Conflicto, resistencia y etnicidad. Revista de Anropología Iberoamericana. 2017; 12(2): 169-192.

58. Velásquez T. The science of corporate social responsibility (CSR): Contamination and conflict in a mining project in the southern Ecuadorian Andes. Resources Policy. 2012; 37(2): 233-240.

59. Sánchez L, Espinosa M, Eguiguren M. Percepción de conflictos socioambientales en zonas mineras: una exploración previa al Proyecto Mirador, Ecuador. Ambiente \& Sociedad. 2016; 19(2).

60. Gallarday T. Estudio del impacto ambiental de la extracción aurífera artesanal dentro de la jurisdicción política del distritito de Santa Rosa de Quives, año 2006. Revista del Instituto de Investigaciones FIGMMG. 2006; 9(18):98-108. 